\title{
Comparison of the characteristics of mesenchymal stem cells obtained from prostate tumors and from bone marrow cultured in conditioned medium
}

\author{
GUANXIONG DING $^{1 *}$, JIALIANG SHAO ${ }^{1 *}$, QIANG DING $^{1}$, ZUJUN FANG $^{1}$, \\ ZHONG WU ${ }^{1}$, JIANFENG XU ${ }^{1,2}$ and PENG GAO ${ }^{1}$ \\ ${ }^{1}$ Department of Urology, Huashan Hospital, Fudan University, Shanghai, P.R. China; \\ ${ }^{2}$ Department of Urology, Wake Forest University School of Medicine, Winston-Salem, NC, USA
}

Received March 20, 2012; Accepted May 31, 2012

DOI: $10.3892 / \mathrm{etm} .2012 .642$

\begin{abstract}
Prostate cancer (PCa) is the most common type of cancer worldwide. Mesenchymal stem cells (MSCs) can also be utilized as 'tumor stromal cells', which are associated with invasive and metastatic malignant tumor cells. Our study aimed to investigate MSCs in prostate tumors and normal MSCs and evaluate their differential characteristics. Normal MSCs (BMMSCs) were isolated from the femur and tibia of normal mice; prostate tumor MSCs (PCa-MSCs) were obtained from prostate tumors implanted in mice. These two types of MSCs were induced to differentiate into adipocytes, bone cells and chondrocytes. Growth curves were used to analyze the growth ability of PCa-MSCs and BMMSCs. Tritium-labeled thymidine (3H-TdR) was used to evaluate cell proliferation of RM-1 stimulated by MSCs. The time taken for PCa-MSCs to reach $90 \%$ confluence was markedly shorter than that of BMMSCs (8-10 vs. 12-14 days). The differentiation ability of PCa-MSCs was similar to that described in previous reports. The growth ability of PCa-MSCs was significantly higher than that of BMMSCs. The proliferative activity of PCa-MSCs was also higher than that of BMMSCs. Our data showed that PCa-MSCs exhibit identical characteristics when compared with those of MSCs. Additionally, their proliferative activity and growth ability were significantly higher when compared with these values in BMMSCs, which appear to have an intrinsic, cell-specific capacity to localize to PCa. The possible role of $\mathrm{PCa}-\mathrm{MSCs}$ in the process of $\mathrm{PCa}$ development requires further clarification.
\end{abstract}

Correspondence to: Dr Peng Gao, Department of Urology, Huashan Hospital, Fudan University, 12 Central Wulumuqi Road, Shanghai 200000, P.R. China

E-mail: gp_fudan@126.com

${ }^{*}$ Contributed equally

Key words: prostate cancer, mesenchymal stem cells, RM-1

\section{Introduction}

Prostate cancer (PCa) is the most common type of cancer worldwide, and still ranks as the leading cause of death among urological malignancies. One in six men will be diagnosed with PCa during their lifetime and approximately 217,000 new cases were diagnosed in the US in 2010 (1). PCa progression involves tumor cell proliferation and infiltration into surrounding tissue and induction of various adaptive pathophysiological and pathomorphological processes in tissues that become involved in the developing tumor stroma. However, the mechanism involved in the development of PCa is still unclear.

Within the bone marrow stroma there exists a subset of nonhematopoietic cells referred to as mesenchymal stem cells (MSCs) (2-4). These cells can be expanded ex vivo and induced in vitro or in vivo to terminally differentiate into osteogenic, adipogenic, chondrogenic, and myogenic lineages under appropriate conditions. In addition, MSCs migrate to sites of injury and inflammation and tumors $(5,6)$. Phenotypically, MSCs are identified by the absence of the CD34 and CD45 hematopoietic cell markers and are positive for Thy-1 (CD90), endoglin (CD105), vascular cell adhesion molecule-1 (VCAM-1/CD106), $\mathrm{SH} 2$ and SH3. MSCs express major histocompatibility complex (MHC) class I but do not express MHC class II, B7-1, B7-2, CD40, or CD40L molecules (7).

MSCs can be utilized as 'tumor stromal cells', targeting invasive and metastatic malignant tumor cells (8). Djouad et al (9) also found that MSCs were associated with side effects related to systemic immunosuppression favoring tumor growth in vivo. It is therefore important to investigate the factors related to the in vivo promotion of tumor growth by MSCs and explore the safety of clinical applications of MSCs. As the microenvironment of $\mathrm{PCa}$ is similar to that of injured/stressed tissue $(10,11)$, it was hypothesized that PCa may provide a conducive environment for the grafting of exogenously administered MSCs (12).

Therefore, in the present study, we aimed to investigate the characteristics of MSCs obtained from bone marrow or prostate tumors. Additionally, we investigated whether the proliferation of grafted MSCs in the developing tumors is capable of generating a significant fraction of tumor stroma. 

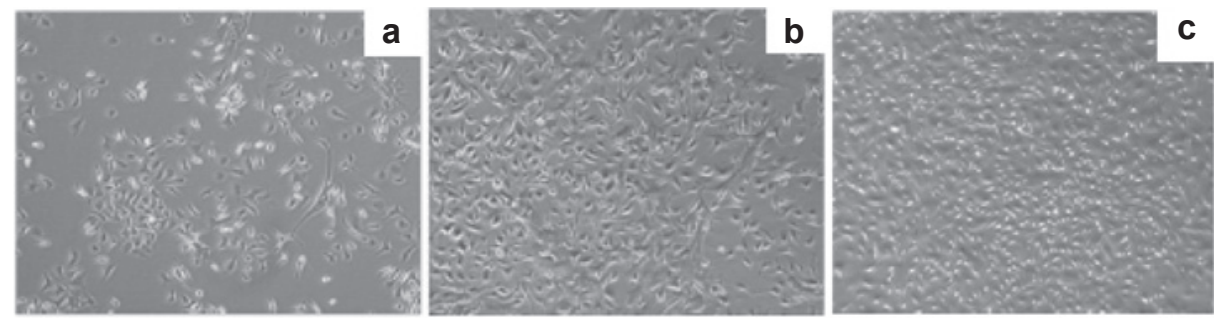

Figure 1. Characterization of isolated PCa-MSCs from the prostate tumors: (a) 48 hours after plating and (b) 7 days after plating as detected using microscopy (magnification, x100). (c) The third generation of PCa-MSCs as detected using microscopy (magnification, x100).
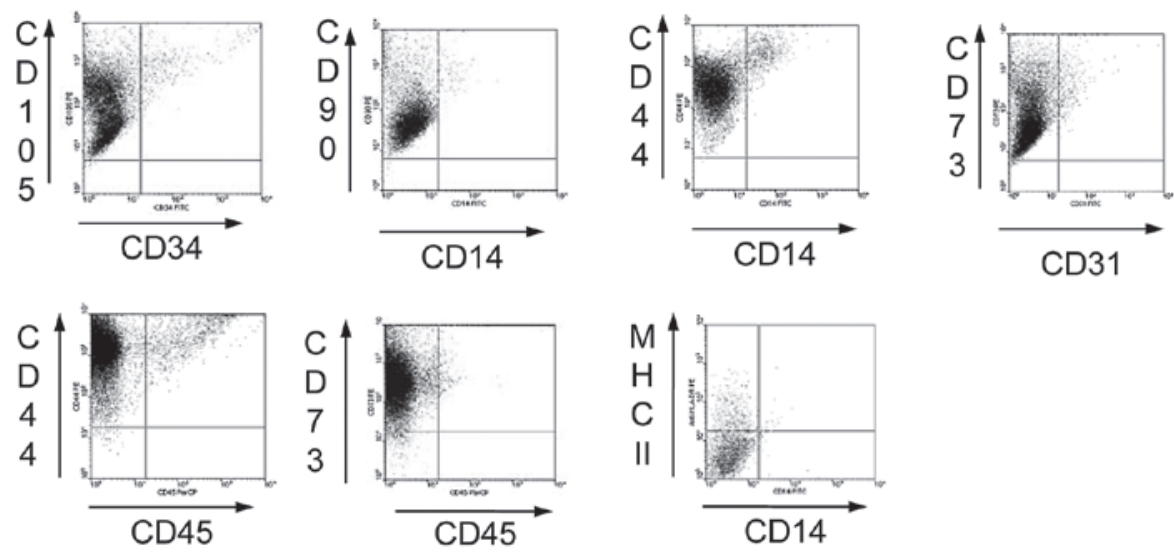

Figure 2. The P3 PCa-MSCs exhibited high expression of CD44, CD73, CD90 and CD105, but were negative for CD14, CD34, CD45 and MHC-II. The PCaMSCs were $95 \%$ homogeneous.

\section{Materials and methods}

Animal subjects. Male mice (nu/nu) were purchased from the Animal Production Area of the Fudan University Cancer Research Center. All animal manipulations were carried out in accordance with Fudan University guidelines under approved protocols. Male four-week-old BALB/c mice $(n=20)$ were maintained and bred under specific pathogen-free conditions, and were divided into the various experimental groups.

Cell lines and animal model. RM-1 cells were purchased from the Institute of Cell Biology, Shanghai, China and cultured in DMEM supplemented with $10 \%$ fetal bovine serum, $100 \mathrm{U} / \mathrm{ml}$ penicillin, $100 \mathrm{~g} / \mathrm{ml}$ streptomycin at $37^{\circ} \mathrm{C}$ in a humidified $5 \% \mathrm{CO}_{2}$ incubator. BALB/c mice $(\mathrm{n}=12)$ inoculated subcutaneously with RM-1 cells were used as a model of prostate cancer whereas the control group comprised $8 \mathrm{BALB} / \mathrm{c}$ mice injected with physiological saline. The time and the efficiency of cancer formation were measured.

Mesenchymal stem cell isolation and culture. The methods described by Peister et al were used (13). Briefly, MSCs were extracted from the femur, tibia and humerus of normal mice (BMMSCs) under axenic conditions by washing with PBS and filtration through a 200-mesh sieve net. Prostate tumors were obtained within 15 days following injection. Tumor tissue isolated from the mice with prostate cancer was cut into $3 \mathrm{~mm}^{3}$ pieces. Prostate tumor MSCs (PCa-MSCs) were obtained after filtration through a 200-mesh sieve net and centrifugation. CD105 cells separated using magnetic beads were cultured in DMEM-LG medium containing 10\% fetal bovine serum.

In vitro efficacy experiments of MSCs. MSCs are progenitors of skeletal tissue components such as bone, cartilage and adipocytes. To ascertain the in vitro differentiation ability of MSCs isolated from bone marrow stroma and prostate tumor we induced differentiation using a previously described method (14-16).

Growth ability of the BMMSCs and PCa-MSCs. The growth ability of the two types of MSCs (BMMSCs vs PCa-MSCs) was compared using growth curves. The RM-1 cell concentration was adjusted to $1 \times 10^{7} / \mathrm{ml}$ with RPMI- 1640 . The RM-1 cells were grown in 96-well culture plates (Nunc Inc.) with $1 \times 10^{6} /$ well density, to which different concentrations $(1: 1,1: 2$, 1:3, 1:4 and 1:5) of BMMSCs or PCa-MSCs were added. The 96-well culture plates were cultured in DMEM/F12 medium $\left(37^{\circ} \mathrm{C}, 5 \% \mathrm{CO}_{2}\right)$. The culture was terminated prior to $12-16$ h by adding $100 \mu \mathrm{l}(0.5-1 \mu \mathrm{Ci})$ tritium labeled thymidine (3H-TdR) to each well. After the end of the culture, the cells were collected on glass fiber filter paper for natural drying. Scintillation counting (cpm) values were determined each minute using a beta liquid scintillation counter. This experiment was also performed with the control group (PBS).

Statistical analysis. All data are analyzed using the SigmaStat statistical software (Jandel Scientific, San Rafael, CA, USA) and S SigmaPlot (SPSS Inc. Chicago, IL, USA). P $<0.05$ was considered to indicate a statistically significant difference. 

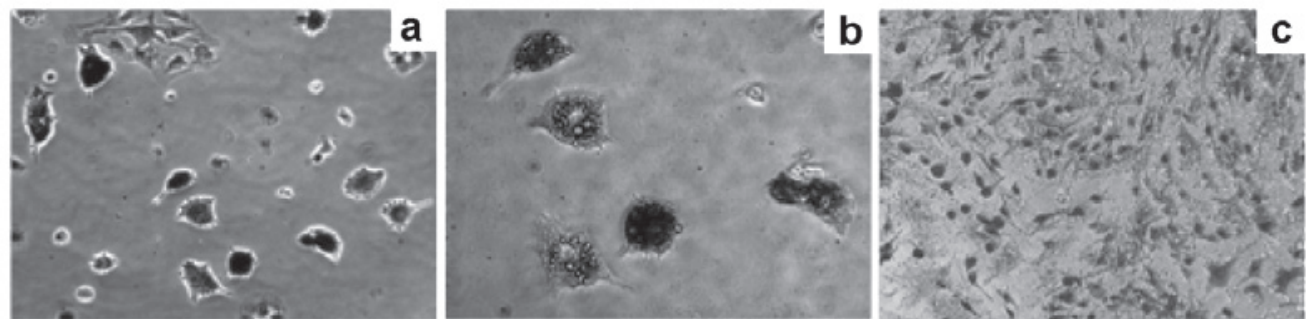

Figure 3. PCa-MSCs were induced to differentiate into adipocytes (Oil red O). (a) The PCa-MSCs exhibiting a long spindle-shape gradually become oval or round at 14 days as detected under microscopy (magnification, x100). (b) Intracytoplasmic refractive bright circular lipid droplets were noted at 14 days under microscopy (magnification, x100). (c) The normal control cytoplasm was not stained by Oil red O.
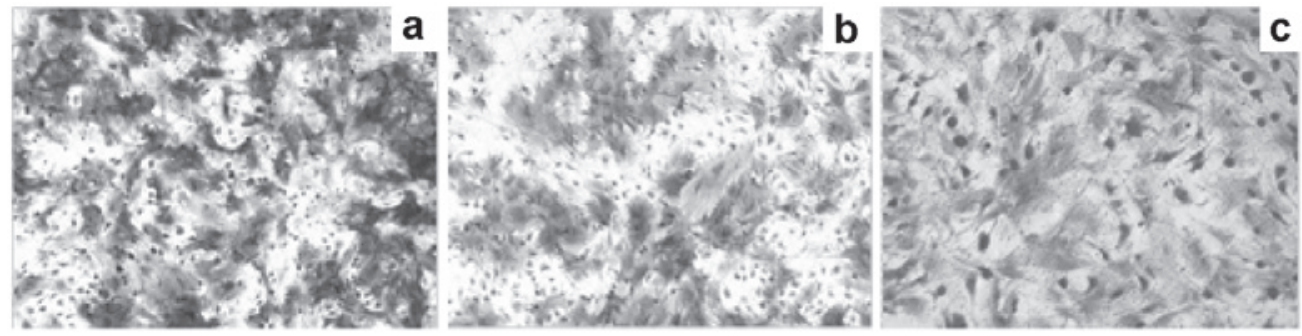

Figure 4. PCa-MSCs were induced to differentiate into bone cells (alkaline phosphatase). (a) The cell morphology of PCa-MSCs changed from a spindleshaped to a flat-shape at 10 days as detected under microscopy (magnification, x40). (b) More red alkaline grain-containing acid enzyme-positive granules in the cytoplasm at 10 days were noted under microscopy (magnification, x100). (c) In the normal controls, alkaline phosphatase expression was almost negative as noted under microscopy (magnification, $\mathrm{x} 100)$.
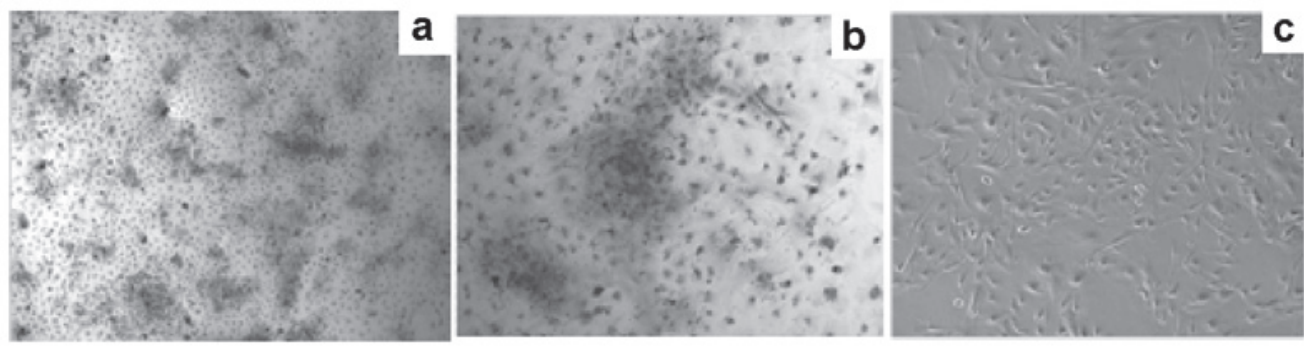

Figure 5. PCa-MSCs were induced to differentiate into bone cells (alizarin red): (a) with $0.1 \%$ alizarin red staining at 14 days under microscopy (magnification, $\mathrm{x} 40$ ). (b) Visible orange-red nodules and a clear boundary of the mineralized nodules were noted at 14 days as detected under microscopy (magnification, x100). (c) The normal control cells showed negativity to alizarin red under microscopy (magnification, x100).
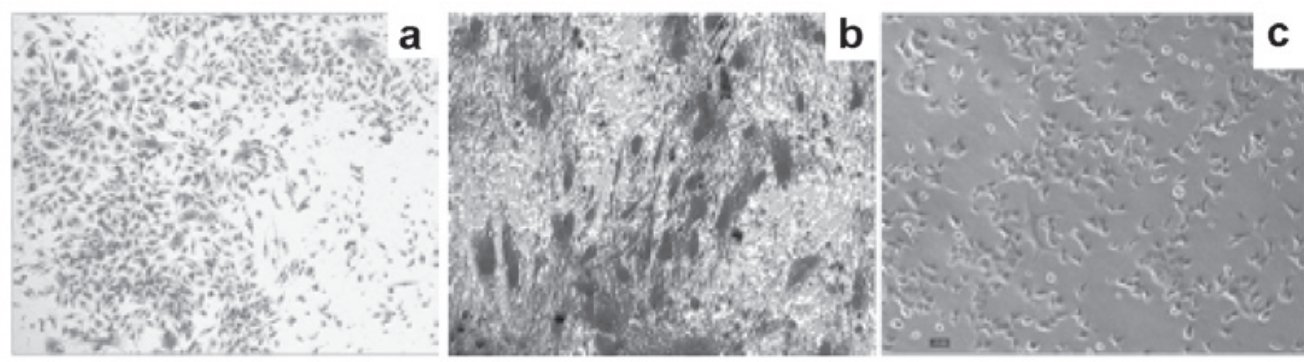

Figure 6. PCa-MSCs were induced to differentiate into chondrocytes (toluidine blue). (a) The PCa-MSCs continued to proliferate to form multiple cell nodules; cells were polygonal or round shape at 10 days as detected under microscopy (magnification, $\mathrm{x} 40$ ). (b) The cells exhibited blue metachromasia in the cytoplasm when stained with toluidine blue at 10 days after induction as detected under microscopy (magnification, x100). (c) The normal control cells were negative as detected under microscopy (magnification, x100).

\section{Results}

Cell culture. The results of the BMMSC culture were similar to those of previous reports (17). We therefore focused on the results for the PCa-MSCs culture. Fig. 1a demonstrates the cell growth status $48 \mathrm{~h}$ after primary vaccination in the 


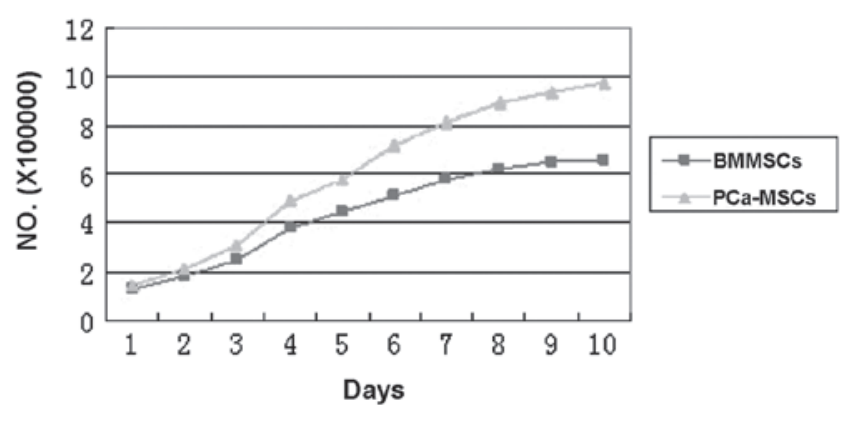

Figure 7. Growth curves of the PCa-MSCs and BMMSCs.

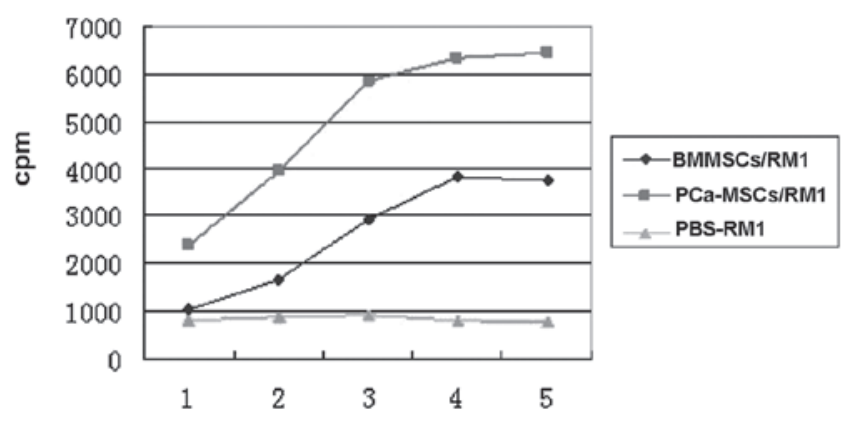

Figure 8. RM1 cell proliferation experiments evaluated by 3H-TdR.

culture medium. We used microscopy to observe the extent of cell growth and the number of colonies, which were fusiform. The time required for $90 \%$ confluence of the PCa-MSCs was markedly shorter than that of the BMMSCs (Fig. 1b; 8-10 days vs. 12-14 days). PCa-MSCs were cultured, often with a mixture of various cells, past 2-3 generations and became uniform in morphology (Fig. 1c). As detected by flow cytometry, the P3 PCa-MSCs exhibited high expression of CD44, CD73, CD90 and CD105, but were negative for CD14, CD34, CD45 and MHC-II. The PCa-MSCs were 95\% homogeneous (Fig. 2).

\section{Differentiation of $\mathrm{PCa}$-MSCs}

PCa-MSCs were induced to differentiate into adipocytes $($ Oil red $O$ ). When induced by adipogenic medium, the PCa-MSCs exhibiting a long spindle-shaped form gradually became oval or round (Fig. 3a), with intracytoplasmic refractive bright circular lipid droplets (Fig. 3b). Lipid droplets exhibited a brick-red color after staining with Oil red $\mathrm{O}$ while the normal control cytoplasm was not stained (Fig. 3c).

PCa-MSCs were induced to differentiate into bone cells (alkaline phosphatase). With osteogenic induction, the cell morphology of PCa-MSCs changed from a spindle-shaped to a flat-shaped morphology (Fig. 4a) and cells were observed to be alkaline phosphatase-positive after 10 days (more red alkaline grain-containing acid enzyme-positive granules in the cytoplasm, Fig. 4b), whereas in the normal controls, alkaline phosphatase expression was almost negative (Fig. 4c).

$P C a-M S C s$ were induced to differentiate into bone cells (alizarin red). The PCa-MSCs exposed to osteogenic induction with $0.1 \%$ alizarin red staining exhibited visible orange-red nodules and a clear boundary of the mineralized nodules after
14 days (Fig. 5a and b), whereas normal control cells showed negativity to alizarin red staining (Fig. 5c).

PCa-MSCs were induced to differentiate into chondrocytes (toluidine blue). After chondrogenic induction, the PCa-MSCs continued to proliferate to form multiple cell nodules, in which cells were polygonal or round in shape (Fig. 6a). We observed that the cells exhibited blue metachromasia in the cytoplasm stained with toluidine blue 10 days after induction (Fig. 6b). The normal control cells were negative (Fig. 6c).

Proliferative activity and growth ability of BMMSCs and $P C a-M S C s$. We found that the growth ability of PCa-MSCs was markedly higher than that of BMMSCs. The growth curve of these two cell types is shown in Fig. 7. Based on the RM-1 cell proliferation experiments we found that the proliferative activity of PCa-MSCs was also higher than that of the BMMSCs (Fig. 8).

\section{Discussion}

In this study, we provide evidence that MSCs home to subcutaneously implanted mouse prostate tumors, which was similar to the results of previous studies, in which cells were observed in the lung and liver. Our methods for PCa-MSC isolation and culture were effective, since the cells showed a phenotype of CD34/CD45- [not hematopoietic cells $(18,19)$ ], $\mathrm{CD}_{4}{ }^{+} / \mathrm{CD} 3^{+} / \mathrm{CD} 105^{+} / \mathrm{CD} 90^{+}$[mesenchymal stromal cell and stem cell markers (20-22)] and CD14/MHC-II- [not endothelial progenitor cells (21-23)]. The isolation of PCa-MSCs from prostate tumors is an important aspect of our study. In fact, to our knowledge, this is the first demonstration of PCa-MSCs obtained from prostate tumors implanted in mice. Alternatively, human $\mathrm{PCa}$, similar to other cancers, requires the elaboration of mesodermal elements, specifically endothelial cells and pericytes. It has been suggested that MSCs are a main source of pericytes within the bone marrow stroma $(24,25)$; thus, MSCs may integrate into prostate cancer to contribute to the mesenchymal elements of the tumor. MSCs may localize to the tumor under physiological conditions to assist with tissue repair. This results in a microenvironment conducive to tumor growth.

Mesenchymal stem cells are a type of primary cell that self-renew and have multiple differentiating potentials (26). BMMSCs differentiate into nerve cells, skeletal muscle cells, and vascular endothelial cells. Our study also provides evidence that PCa-MSCs have differentiating ability, which is consistent with other reports (14-16,17). Collectively, these findings suggest that in the process of prostate cancer development, MSCs may confer a potential therapeutic advantage against bone metastases in PCa.

One aim of this study was to assess the proliferative activity and growth ability of PCa-MSCs compared with BMMSCs. To achieve this aim, we used cell proliferation and MSC-RM-1 cell culture. The growth curve indicated that the growth ability of PCa-MSCs was markedly higher than that of BMMSCs. In addition, the activity of PCa-MSCs, which could stimulate the cell proliferation of RM-1, was significantly higher when compared with that of BMMSCs. Our results indicate that it may be mediated at least in part by growth factors/chemokines. This observation is consistent with the 
hypothesis that MSCs locate to the tumor environment since tumors mimic tissue injury $(10,11,27)$. Conversely, MSCs are precursors of stromal cells, which generate the extracellular matrix supporting hematopoiesis within the bone marrow microenvironment (28). Stromal components derived from MSCs may therefore play a role in tumor growth within the tumor microenvironment.

Taking these findings together, it is unlikely that the localization of PCa-MSCs within prostate tumors grown in mice was merely the result of a species-specific tropism. Instead, MSCs appear to have an intrinsic, cell-specific capacity to localize to PCa. Detailed characterization of the properties of MSCs following tumor grafting will be addressed in future studies.

\section{Acknowledgements}

This study was sponsored by a grant from the National Natural Sciences Foundation of China (NSFC) (grant no. 81001145).

\section{References}

1. Richman EL, Kenfield SA, Stampfer MJ, Paciorek A, Carroll PR and Chan JM: Physical activity after diagnosis and risk of prostate cancer progression: data from the cancer of the prostate strategic urologic research endeavor. Cancer Res 71: 3889-3895, 2011.

2. Pittenger MF and Marshak DR: Mesenchymal stem cells of human adult bone marrow. In: Stem Cell Biology. Marshak DR, Gardner RL and Gottlieb D (eds). Cold Spring Harbor Laboratory Press, Cold Spring Harbor; pp349-373, 2001

3. Orkin SH: Hematopoietic stem cells: molecular diversification and developmental interrelationships. In: Stem Cell Biology. Marshak DR, Gardner RL and Gottlieb D (eds). Cold Spring Harbor Laboratory Press, Cold Spring Harbor; pp289-306, 2001.

4. Keller G: The hemangioblast. In: Stem Cell Biology. Marshak DR, Gardner RL and Gottlieb D (eds). Cold Spring Harbor Laboratory Press, Cold Spring Harbor; pp329-348, 2001.

5. Kopen GC, Prockop DJ and Phinney DG: Marrow stromal cells migrate throughout forebrain and cerebellum, and they differentiate into astrocytes after injection into neonatal mouse brains. Proc Natl Acad Sci USA 96: 10711-10716, 1999.

6. Woodbury DJ, Schwarz EJ, Prockop DJ and Black IB: Adult rat and human bone marrow stromal cells differentiate into neurons. J Neurosci 61: 364-370, 2000.

7. Noël D, Djouad F and Jorgensen C: Regenerative medicine through mesenchymal stem cells for bone and cartilage repair Curr Opin Investig Drugs 3: 1000-1004, 2002.

8. Nakamura K, Ito Y, Kawano Y, Kurozumi K, Kobune M, Tsuda H, Bizen A, Honmou O, Niitsu Y and Hamada H: Anti-tumor effect of genetically engineered mesenchymal stem cells in a rat glioma model. Gene Ther 11: 1155-1164, 2004

9. Djouad F, Plence P, Bony C, Tropel P, Apparailly F, Sany J, Noel D and Jorgensen C: Immunosuppressive effect of mesenchymal stem cells favors tumor growth in allogeneic animals. Blood 102: 3837-3844, 2003.

10. Bissell MJ and Radisky D: Putting tumors in context. Nat Rev Cancer 1: 46-54, 2001.
11. Ben-Baruch A: Host microenvironment in breast cancer development: inflammatory cells, cytokines and chemokines in breast cancer progression: reciprocal tumor-microenvironment interactions. Breast Cancer Res 5: 31-36, 2003.

12. Studeny M, Marini FC, Champlin RE, Zompetta C, Fidler IJ and Andreeff M: Bone marrow-derived mesenchymal stem cells as vehicles for interferon-beta delivery into tumors. Cancer Res 62: 3603-3608, 2002.

13. Peister A, Mellad J, Larson B, Hall B, Gobson L and Prockop D: Adult stem cells from bone marrow (MSCs) isolated from different strains of in bred mice vary in surface epitopes, rates of proliferation, and differential potential. Blood 103: 1662-1668, 2004.

14. Igarashi M, Yogiashi Y, Mihara M, Takada I, Kitagawa H and Kato S: Vitamin K induces osteoblast differentiation through pregnane $\mathrm{X}$ receptor-mediated transcriptional control of the Msx2 gene. Mol Cell Biol 27: 7947-7954, 2007.

15. Meirelles Lda S and Nardi NB: Murine marrow-derived mesenchymal stem cell: isolation, in vitro expansion, and characterization. Br J Haematol 123: 702-711, 2003

16. Phinney DG, Kopen G, Isaacson RL and Prockop DJ: Plastic adherent stromal cells from the bone marrow of commonly used strains of inbred mice: variations in yield, growth, and differentiation. J Cell Biochem 72: 570-585, 1999.

17. Pittenger MF, Mackay AM, Beck SC, Jaiswal RK, Douglas R, Mosca JD, Moorman MA, Simonetti DW, Craig S and Marshak DR: Multilineage potential of adult human mesenchymal stem cells. Science 284: 143-147, 1999.

18. Huss R, Lange C, Weissinger EM, Kolb HJ and Thalmeier K: Evidence of peripheral blood-derived, plastic-adherent CD34(-/low) hematopoietic stem cell clones with mesenchymal stem cell characteristics. Stem Cells 18: 252-260, 2000.

19. Majumdar MK, Banks V, Peluso DP and Morris EA: Isolation, characterization, and chondrogenic potential of human bone marrow-derived multipotential stromal cells. J Cell Physiol 185: 98-106, 2000

20. De Ugarte DA, Alfonso Z, Zuk PA, Elbarbary A, Zhu M, Ashiian P, Benhaim P, Hedrick MH and Fraser JK: Differential expression of stem cell mobilization-associated molecules on multi-lineage cells from adipose tissue and bone marrow. Immunol Lett 89: 267-270, 2003.

21. Niyibizi C, Wang S, Mi Z and Robbins PD: The fate of mesenchymal stem cells transplanted into immunocompetent neonatal mice: implications for skeletal gene therapy via stem cells. Mol Ther 9: 955-963, 2004.

22. Majumdar MK, Thiede MA, Mosca JD, Moorman M and Gerson SL: Phenotypic and functional comparison of cultures of marrow-derived mesenchymal stem cells (MSCs) and stromal cells. J Cell Physiol 176: 57-66, 1998.

23. Phinney DG: Building a consensus regarding the nature and origin of mesenchymal stem cells. J Cell Biochem Suppl 38: 7-12, 2002.

24. Conget PA and Minguell JJ: Phenotypical and functional properties of human bone marrow mesenchymal progenitor cells. J Cell Physiol 181: 67-73, 1999.

25. Minguell JJ, Erices A and Conget P: Mesenchymal stem cells. Exp Biol Med 226: 507-520, 2001.

26. Huttmann A, Li CL and Duhrsen U: Bone marrow-derived stem cells and 'plasticity'. Ann Hematol 82: 599-604, 2003.

27. Coussens LM and Werb Z: Inflammation and cancer. Nature 420: 860-867, 2002.

28. Prockop DJ: Marrow stromal cells as stem cells for nonhematopoietic tissues. Science 276: 71-74, 1997. 